Pacific Journal of Mathematics

ON THE LORIMER-RAHILLY AND JOHNSON-WALKER 


\title{
ON THE LORIMER-RAHILLY AND JOHNSON-WALKER TRANSLATION PLANES
}

\author{
ViKRAM JHA AND MichaEL J. KALLAHER
}

\begin{abstract}
We investigate finite translation planes of dimension $d$ over the kernel $K=G F(q)$, where $q=p^{k}$ with $p$ a prime, having a collineation group $G$ with either $G=$ PSL $(2, w)$ or $G=\mathbf{S L}(3, w)$, where $w$ is a prime power. We derive several restrictions on the planes; for example, if $p$ is odd then 4 divides $d$. We also give a new characterization of the Lorimer-Rahilly and Johnson-Walker planes of order 16, which is more general than that of Johnson and Ostrom. In addition, we give many examples indicating how good are our results.
\end{abstract}

1. Introduction. Lorimer [16] constructed an interesting translation plane of order 16 . The interesting aspect of this plane is that it possesses the group $\operatorname{SL}(3,2)=\operatorname{PSL}(2,7)$ as a collineation group; in fact, the linear translation complement contains the group SL $(3,2)=$ PSL $(2,7)$ as a subgroup. Rahilly [21] independently constructed a translation plane of order 16 which was tangentially transitive in the sense of Jha [11]. Johnson and Ostrom [12] showed that the Lorimer and Rahilly planes are the same plane; we will call their translation plane the Lorimer-Rahilly translation plane. Walker [20] in a note on tangentially transitive planes gave a different construction of the Lorimer-Rahilly plane and, in addition, showed that this construction gives a second translation plane, distinct from the Lorimer-Rahilly plane, of order 16 which also possesses SL $(3,2)=$ PSL $(2,7)$ as a subgroup of the linear translation complement. This second plane was also discovered independently by Johnson; we will call this plane the Johnson-Walker translation plane.

The Lorimer-Rahilly and Johnson-Walker translation planes have the following common properties: (1) Order 16 with Kernel $K=$ $G F(2)$ and hence dimension $d=4$; (2) The linear translation complement contains a subgroup $G=\mathrm{SL}(3,2)=\operatorname{PSL}(2,7)$ having the following action on the line at infinity: The group $G$ fixes pointwise a set $\Delta$ of 3 points and is transitive on the remaining 14 points.

These observations led the authors to investigate the following questions: Which translation planes have a collineation group $G=$ PSL $(2, w)$ for some prime power $w$ ? Which translation planes have a collineation group $G=\mathrm{SL}(3, w)$ for some prime power $w$ ?

Our main results are as follows. Let $\pi$ be a finite translation 
plane of dimension $d$ over its kernel $K=G F(q)$, where $q=p^{k}$ with $k \geqq 1$ and $p$ a prime.

I. Assume $G=\operatorname{PSL}(2, w)$, where $w \geqq 3$, is a subgroup of the linear translation complement $L C(\pi)$ of $\pi$.

A. If $p$ is odd then 4 divides $d$.

B. If $p=2$ and $w \geqq 7$ then 2 divides $d$.

C. If $w=p^{s}$ for some $s \geqq 1$, and if $G$ fixes pointwise a subset $\Delta$ of the line at infinity $\ell_{\infty}$ with $|\Delta|=p^{t}+1$ for some $t \geqq 1$ and is transitive on $\ell_{\infty}-\Delta$, then $p=2$.

II. Assume $G=\mathrm{SL}(3, w)$, where $w \geqq 2$, is a subgroup of the linear translation complement $L C(\pi)$ of $\pi$.

A. Either $p$ is odd and 4 divides $d$, or $p=2$ and 2 divides $d$.

B. If $w=p^{t}$ with $t \mid k$ and if $G$ leaves invariant a subset $\Delta$ of $\iota_{\infty}$ with $|\Delta|=w+1$ and is transitive on $\iota_{\infty}-\Delta$, then

(i) $d=4$ and $w=q$

(ii) $G$ fixes $\Delta$ pointwise

(iii) $p=2$.

C. If $w=p$ and if $G$ leaves invariant a subset $\Delta$ of $\ell_{\infty}$ with $|\Delta|=p+1$ and is transitive on $\ell_{\infty}-\Delta$, then $\pi$ is either the Lorimer-Rahilly translation plane or the Johnson-Walker translation plane.

In [14] Johnson and Ostrom gave a characterization of the Lorimer-Rahilly and Johnson-Walker planes as the only translation planes of order 16 having SL $(3,2)=\operatorname{PSL}(2,7)$ acting on them. Our characterization of the same planes in II C is more general since we do not limit the order of the plane to 16 or the value of $w$ to 2 , but instead we characterize the planes in terms of the action of the group SL $(3, w)$ on the line $\ell_{\infty}$.

In addition, throughout the article many examples are given to indicate in what ways these results are best possible. For example, with relation to the results given in I the group PSL $(2,2)=S_{3}$ occurs in many translation planes; thus the condition " $w \geqq 3$ " is a necessary one. On the other hand, it appears that some of the assumptions could be dropped without seriously affecting the conclusions. Lorimer has raised the question: "Which translation planes possess a collineation group $G=\mathrm{SL}(3, q)$, where $q$ is the order of the kernel?" The results of this article indicate that there are only a small number of such translation planes.

The authors would like to thank the referee for his help; in particular, he corrected a major error in the proof of Theorem 3.6.

2. Preliminaries. We assume the reader is familiar with the theory of projective planes as given in Dembowski [2] and Ostrom [19] and with the basics of group theory as given in Huppert [10] 
In this section we give the viewpoint of (affine) translation planes that will be taken in this article. This viewpoint, which is originally due to Johannes André, has been developed by T. G. Ostrom in many of his recent articles. (See Ostrom [19, 20].) Most of the definitions and results stated here can be found in the article [15].

All planes that we consider will have finite order. Furthermore, all planes will be (affine) translation planes and the collineations considered will be in the translation complement of the plane. The translation complement is defined as follows (see [15]): Let $\pi$ be a translation plane and let $G$ be its collineation group. It follows that $G=T G_{0}$ where $T$ is the group of translations of $\pi$ and $G_{0}$ is the subgroup of $G$ fixing an affine point $\mathcal{O}$ of $\pi$. The point $\delta$ is usually taken to be the origin of a coordinate system for $\pi$ with respect to a quasi-field $(Q,+, \cdot)$. The group $C(\pi)=G_{0}$ is called the translation complement (with respect to $\mathcal{O}$ ) of $\pi$ and a line through $\sigma$ is called a component of $\pi$.

The kernel of the translation $\pi$ is the field $K=K(\pi)=\{k \mid k \in Q$, $k(a b)=(k a) b$ and $k(a+b)=k a+k b$ for all $a, b \in Q\}$. Geometrically, the group of $\left(\mathcal{O}, \ell_{\infty}\right)$-homologies is isomorphic to the multiplicative group $K^{*}$ of the kernel $K$ and we will use $K^{*}$ to denote this group. (Here, and throughout this article, the line at infinity of the translation plane is denoted by $\ell_{\infty}$.) Concerning these concepts we have the following information.

THEOREM 2.1. Let $\pi$ be a finite translation plane of order $p^{r}$ where $p$ is a prime and $r \geqq 1$, let $K=G F(q)$ with $q=p^{k}$ be the kernel of $\pi$, and let $C=C(\pi)$ be the translation complement of $\pi$. The following statements hold:

(i) Every component $l$ of $\pi$ is a vector space over $K$ of dimension $d \geqq 1$.

(ii) The plane $\pi$ is a vector space over $K$ of dimension $2 d$.

(iii) The group $C$ is a subgroup of $\Gamma L(2 d, q)$.

(iv) If $l$ is a component of $\pi$, then the group $C_{l}$ induces a group of semi-linear transformations on $l$ which is a subgroup of $\Gamma L(d, q)$.

Proof. This is well known. See, for example, André [1] or Ostrom [19].

REMARK 2.1. Throughout this article, given a translation plane $\pi$ of finite order the characteristic of $\pi$ will always be denoted by the letter $p$-hence $p$ will always be a prime-and the letter $q$ will always denote the order of the kernel $K(\pi)$ of $\pi$. Thus $q=p^{k}$ for some integer $k \geqq 1$. 
DEFINITION 2.1. Let $\pi$ be a finite translation plane of order $p^{r}$ and let $K=G F(q)$, where $q=p^{k}$, be its kernel. The dimension of $\pi$ over $K$ is $d$, where $d$ is the dimension of a component of $\pi$ over $K$. The linear translation complement of $\pi$ is the group $L C(\pi)=$ $C(\pi) \cap G L(2 d, q)$, where $C(\pi)$ is the translation complement of $\pi$.

THEOREM 2.2. If $\mathfrak{A}$ is a finite translation plane of odd dimension $d$ over its kernel $K=G F(q)$, where $q=p^{k}$ with $k \geqq 1$, and if $G$ is a collineation group of $\mathfrak{A}$ fixing an affine line $l$ of $\mathfrak{A}$, then the group $G$ is solvable.

\section{Proof. See Lemma 2.13 of Kallaher and Ostrom [15].}

Definition 2.2. Let $\mathfrak{A}$ be a translation plane. An affine perspectivity (elation, homology) is a perspectivity (elation, homology) whose axis is an affine line of $\mathfrak{A}$. If $\sigma$ is an affine homology contained in the translation complement $C(\mathfrak{A})$, then the co-center of $\sigma$ is the point $l \cap l_{\infty}$, where $l$ is the axis of $\sigma$, and the co-axis of $\sigma$ is the component $m=\mathscr{O} P$, where $P$ is the center of $\sigma$. (Recall that $P$ must be on $l_{\infty}$, the line at infinity.)

LEMMA 2.1. Let $\pi$ be a translation plane of order $p^{r}$ and let $H$ be a group of affine homologies in the translation complement $C(\pi)$ with the same axis (and hence center). The Sylow 2-subgroups of $H$ are cyclic or generalized quaternion; in particular, $H$ has no subgroup isomorphic to the Klein four-group.

Proof. This follows from the fact that $H$ is a Frobenius complement on the co-axis of $H$. (See Lemma 2.2 of Kallaher and Ostrom [15] and Hauptstatz V.8.7 in Huppert [10].

LEMMA 2.2. Let $\pi$ be a finite translation plane of order $p^{r}$ and assume $\pi$ has dimension $d$ over its kernel $K=G F(q)$ with $q=p^{k}$. If $\alpha$ is a Baer involution in the linear translation complement $L C(\pi)$ of $\pi$ and if $\pi_{0}$ is the associated Baer subplane, then the following statements hold:

(i) The subplane $\pi_{0}$ is a subspace of dimension $d$ in $\pi$ considered as a vector space over $K$.

(ii) The subplane $\pi_{0}$ is a translation plane with $K$ contained in its kernel.

Proof. Since $\pi_{0}$ consists precisely of the points of $\pi$ fixed by $\alpha$ it is clear that $\pi_{0}$ forms a subspace of $\pi$ in $K$. The dimension is $d$ since $\pi_{0}$ has $q^{d}$ points. This proves statement (i). For statement 
(ii) note that $\pi_{0}$ a subspace implies $\pi_{0}$ is closed under vector addition. Every translation of $\pi$ has the form

$$
(x, y) \longrightarrow(x, y)+(a, b)
$$

relative to the vector space structure of $\pi$, where $(a, b)$ is a fixed point of $\pi$ and $(x, y)$ is a general point. It is clear that the translation with $(a, b) \in \pi_{0}$ preserves $\pi_{0}$ and hence is a translation of $\pi_{0}$. Thus $\pi_{0}$ has $q^{d}$ translations and hence $\pi_{0}$ is a translation plane. The fact that $K$ is contained in the kernel of $\pi_{0}$ is straightforward to prove.

LEMMA 2.3. Let $V$ be a vector space of dimension $2 d$ over the field $K=G F(q)$, where $q$ is an odd prime power, and let $\alpha, \beta$ be linear transformations of $V$ with $\alpha \beta=\beta \alpha$ and $\alpha^{2}=\beta^{2}=1$. Assume that $V(\alpha)$, the subspace consisting of the fixed points of $\alpha$, and $V(\beta)$, the subspace consisting of the fixed points of $\beta$, both have dimension $d$ over $K$. If $V(\alpha) \cap V(\beta)$ consists only of 0 then $\alpha \beta$ is the scalar linear transformation $\sigma(-1): v \rightarrow(-1) v$ on $V$.

Proof. We have $V=V(\alpha) \oplus V(\beta)$. Since $\alpha \beta=\beta \alpha$, the linear transformation $\alpha$ fixes $V(\beta)$ and thus it induces a linear transformation $\bar{\alpha}$ on $V(\beta)$ with $\bar{\alpha}^{2}=1$. Since $V(\alpha) \cap V(\beta)=0$, the only eigenvalue for $\bar{\alpha}$ is -1 . Thus, by choosing a suitable basis $\bar{B}$ for $V(\beta)$, the transformation $\bar{\alpha}$ is represented by the matrix $(-1) I$, where $I$ is the $d$ by $d$ identity matrix. (Here we are using Theorem 6 on p. 204 of [9].) Similarly, by choosing a suitable basis $B$ for $V(\alpha)$ the linear transformation $\beta$ induces a linear transformation $\bar{B}$ on $V(\alpha)$ which is represented by $(-1) I$ with respect to $B$. Then $B \cup \bar{B}$ is a basis for $V$ and, with respect to this basis, we have the representations

$$
\alpha \longleftrightarrow\left[\begin{array}{cc}
I & 0 \\
0 & (-1) I
\end{array}\right] \quad \beta \longleftrightarrow\left[\begin{array}{cc}
(-1) I & 0 \\
0 & I
\end{array}\right] .
$$

It follows that the transformation $\alpha \beta$ is represented by the matrix

$$
\left[\begin{array}{cc}
(-1) I & 0 \\
0 & (-1) I
\end{array}\right]
$$

with respect to $B \cup \bar{B}$. It follows that $\alpha \beta=\sigma(-1)$.

LEMMA 2.4. Let $\pi$ be a finite translation plane of dimension $d$ over its kernel $K=G F(q)$, where $q=p^{k}$ with $k \geqq 1$ and $p$ is an odd prime. Let $\alpha_{1}, \alpha_{2}$ be two distinct Baer involutions contained in the linear translation complement $L C(\pi)$ and let $\pi_{1}, \pi_{2}$ be the associ- 
ated Baer subplanes of $\alpha_{1}, \alpha_{2}$ respectively. Assume that $\alpha_{1} \alpha_{2}=\alpha_{2} \alpha_{1}$ and that for $i \neq j$ the involution $\alpha_{i}$ induces an involutory homology $\bar{\alpha}_{i}$ on $\pi_{j}$. The collineation $\alpha_{1} \alpha_{2}$ is an involutory homology on $\pi$.

Proof. By Corollary 4 of Foulser [5] we must have $\pi_{1} \neq \pi_{2}$. For $i=1,2$ let $\pi_{i} \cap l_{\infty}$ be the points at infinity of $\pi_{i}$. That is, $\pi_{i} \cap l_{\infty}$ is the line at infinity of $\pi_{i}$. We consider two cases: (a) $\pi_{1} \cap$ $l_{\infty}$ is the axis of $\bar{\alpha}_{2}$, and (b) $\bar{\alpha}_{2}$ has affine axis in $\pi_{1}$.

If case (a) holds, then it follows that the involutory homology $\bar{\alpha}_{1}$ induced on $\pi_{2}$ must have axis $\pi_{2} \cap l_{\infty}$ and that $\pi_{1} \cap l_{\infty}=\pi_{2} \cap l_{\infty}$. Thus the affine Baer subplanes $\pi_{1}$ and $\pi_{2}$ have trivial intersection. Hence we can apply Lemmas 2.2 and 2.3. Therefore, in case (a) the collineation $\alpha_{1} \alpha_{2}$ is the involutory homology with axis $l_{\infty}$ and center $\mathcal{O}$.

If case (b) holds, then there exists a component $l$ of $\pi$ such that $\pi_{1} \cap l$ is the axis of $\bar{\alpha}_{2}$. (Remember that $\alpha_{2}$ fixes the origin $\mathcal{O}=(0,0)$.) But then $\pi_{1} \cap l$ is in $\pi_{2}$ and hence $\bar{\alpha}_{1}$ must fix $\pi_{1} \cap l$ pointwise in $\pi_{2}$. It follows that $\pi_{1} \cap l=\pi_{2} \cap l$. Also $\pi_{1} \cap l$ is a subspace of $l$ considered as a vector space over $K$. Since $\pi_{1}$ is a Baer subplane of $\pi$, the dimension of $\pi_{1} \cap l$ is half that of $l$; thus, the subspace $\pi_{1} \cap l$ has dimension $(1 / 2) d$ since $l$ has dimension $d$. The set $H=\left\{1, \alpha_{1}, \alpha_{2}, \alpha_{1} \alpha_{2}\right\}$ induces a group of linear transformations on $l$. By Maschke's theorem (Theorem 2.3 and Exercise 2 on p. 25-26 of Dixon [4]), we have $l=\left(\pi_{1} \cap l\right) \oplus L$ where $L$ is a subspace of $l$ on which both $\alpha_{1}$ and $\alpha_{2}$ are fixed-point-free. It follows that both $\alpha_{1}$ and $\alpha_{2}$ are represented on $l$ by the same matrix

$$
\left[\begin{array}{cc}
I & 0 \\
0 & (-1) I
\end{array}\right]
$$

with respect to a suitable basis. (Here we use Theorem 6 on p. 204 and Theorem 8 on p. 207 of [9].) Then $\alpha_{1} \alpha_{2}$ is represented on $l$ by the identity matrix; it follows that $\alpha_{1} \alpha_{2}$ induces the identity on $l$. Therefore, in case (b) the collineation $\alpha_{1} \alpha_{2}$ is an involutory homology with a component as axis.

We close this section with an important result which will be essential in the next two sections. This result is originally due to Professor T. G. Ostrom.

TheOREM 2.3. Let $\pi$ be a finite translation plane of dimension $d$ over its kernel $K=G F(q)$, where $q=p^{k}$ with $k \geqq 1$ and $p$ an odd prime. If the linear translation complement $L C(\pi)$ of $\pi$ contains a Klein four-group $H$ in which all the involutions are mutually conjugate in $L C(\pi)$, then 4 divides $d$ and the involutions in $H$ are Baer involutions. 
Proof. Assume first that $H$ contains an involutory homology. Then all three involutions of $H$ are homologies. If these involutory homologies did not have the same axis or the same center, then the three axes would form the sides of a triangle, one of which is the line at infinity (Dembowski $[2 ; 3.1 .7]$ ). But then the line at infinity must be moved - a contradiction. If the three involutions of $H$ have the same axis then they have the same center since $H$ has no nontrivial elation. Similarly, if they have the same center then they have the same axis. Then, let $l$ be the common axis of the three involutory homologies in $H$. Either $l$ is the line at infinity or $l$ is an affine line of $\pi$. Since $\pi$ has exactly one involutory homology in $C(\pi)$ with axis $l_{\infty}$ (Dembowski $[2,4.3 .10]$ ), the line $l$ must be an affine line. But Lemma 2.1 says we have a contradiction. It follows that all the involutions of $H$ are Baer involutions.

Let $\alpha_{1}, \alpha_{2}, \alpha_{3}=\alpha_{1} \alpha_{2}$ be the Baer involutions in $H$ and let $\pi_{1}, \pi_{2}$, $\pi_{3}$ be the corresponding "Baer exes" (i.e., the Baer subplanes of $\pi$ fixed pointwise by $\alpha_{1}, \alpha_{2}, \alpha_{3}$ respectively). Since $p$ is odd, Corollary 4 of Foulser [5] says that $i \neq j$ implies $\pi_{i} \neq \pi_{j}$. Since $H$ is abelian the involution $\alpha_{i}$ fixes the Baer subplane $\pi_{j}$ for all $i$ and for all $j$. Thus, if $i \neq j$ we have two possibilities: (a) the involution $\alpha_{i}$ induces a Baer involution on $\pi_{j}$, and (b) the involution $\alpha_{i}$ induces an involutory homology on $\pi_{j}$. If (a) occurs for some $i$ and $j$ then $\pi_{j}$ must have even dimension over $K$. (See Lemma 2.14 of [15].) Since $\pi_{j}$ has dimension (1/2)d over $K$, the theorem holds in case (a). Thus we may assume (b) holds for all $i, j$ with $i \neq j$. By Lemma 2.4 the involution $\alpha_{3}=\alpha_{1} \alpha_{2}$ must be an involutory homology which is a contradiction. Thus case (b) can not hold. This proves the theorem.

REMARK 2.2. We will apply Theorem 2.3 in each of the next two sections. It will be clear that Theorem 2.3 applies to many of the classical groups including $S_{n}, A_{n}$, PSL $(2, w)$, and SL $(n, w)$.

REMARK 2.3. Theorem 2.3 does not hold for $p=2$ since in this case the possibility exists that the involutions of the Klein fourgroup $H$ are elations with the same affine axis. This can happen, as can be seen by looking at a semi-field plane (desarguesian or non-desarguesian) of characteristic 2 .

3. The group PSL $(2, w)$ as a collineation group. In this section we investigate translation planes having the group $\operatorname{PSL}(2, w)$, where $w$ is a prime power, as a collineation group. The following result shows that we may in essence restrict ourselves to the linear translation complement. 
LEMMA 3.1. Let $\pi$ be a finite translation plane of dimension d over its kernel $K=G F(q)$, where $q=p^{k}$ with $k \geqq 1$ and $p$ an odd prime. If $\pi$ has a collineation group $G=$ PSL $(2, w)$ in its translation complement $C(\pi)$, then one of the following statements holds:

(i ) $w \geqq 4$ and $L C(\pi)$ contains the subgroup $G=\operatorname{PSL}(2, w)$.

(ii) $w=3$ and either $L C(\pi)$ contains a Klein four-group or $L C(\pi)$ contains the subgroup $G=\operatorname{PSL}(2,3)$.

(iii) $w=2$ and either $L C(\pi)$ contains a cyclic subgroup of order 3 or $L C(\pi)$ contains the subgroup $G=$ PSL $(2,2)$.

Proof. Recall that $C(\pi)$ is a subgroup of $\Gamma L(2 d, q)$ and $L C(\pi)=$ $C(\pi) \cap G L(2 d, q)$. Now $\Gamma L(2 d, q) / G L(2 d, q)$ is isomorphic to $\operatorname{Aut}(K)$ and hence is cyclic. Thus $C(\pi) / L C(\pi)$ is a (cyclic) subgroup of Aut $(K)$. Thus, the factor group $G / G \cap L C(\pi)$ is a cyclic group. Now PSL $(2, w)$ is never cyclic for $w \geqq 2$. If $w \geqq 4$, the group PSL $(2, w)$ is simple. Hence, if $w \geqq 4$, then $G \cap L C(\pi)=G$. If $w=$ 3 then PSL $(2,3)=A_{4}$ has three normal subgroups: $1, A_{4}$ and its Sylow 2 subgroup $S$, which is a Klein four-group. It follows that either $G \cap L C(\pi)=S$ or $G \cap L C(\pi)=G$. If $w=2$ then PSL $(2,3)=$ $S_{3}$ and a similar argument shows that $G \cap L C(\pi)$ is cyclic of order 3 or $G \cap L C(\pi)$.

REMARK 3.1. A reasonable question to ask is the following: What occurs if the translation plane $\pi$ of Lemma 3.1 has a collineation group $G=\operatorname{PSL}(2, w)$ for some prime power $w$ but $G$ is not a subgroup of the translation complement $C(\pi)$ ? The answer is that if $w \geqq 4$ or if $p \geqq 5$ then $C(\pi)$ contains a subgroup $G_{1}=\operatorname{PSL}(2, w)$. For, if $G(\pi)$ is the full collineation group of $\pi$ then

$$
G(\pi)=T C(\pi),
$$

where $T$ is the group of translations of $\pi$, and

$$
T \cap C(\pi)=1, G(\pi) / T=C(\pi) .
$$

If $w \geqq 4$ then PSL $(2, w)$ is simple and nonsolvable; hence $T \cap G=1$ since $T$ is elementary abelian of order $q^{2 d}$. Thus $C(\pi)=G(\pi) / T$ contains a subgroup $G_{1}=\operatorname{PSL}(2, w)$. If $w=3$ then either $T \cap G=1$ or $T \cap G=S$, the unique Klein four-group of PSL $(2,3)=A_{4}$; hence $w=3$ implies either $C(\pi)$ contains a subgroup $G_{1}=$ PSL $(2,3)$ or $p=2$. Similarly, if $w=2$ then $\operatorname{PSL}(2,2)=S_{3}$ and hence either $C(\pi)$ contains a subgroup $G_{1}=\operatorname{PSL}(2,2)$ or $p=3$.

Furthermore, in the latter two cases it is not difficult to construct examples where $G(\pi)$ contains a group $G=$ PSL $(2, w)$ with $G \cap T \neq 1$. For instance, consider any translation plane of order 
$q^{d}$ with $q$, the order of the kernel, even and $q-1$ divisible by 3 .

Lemma 3.2. The group $G=\operatorname{PSL}(2, w)$, where $w \geqq 3$, contains a Klein four-group $H$ in which the involutions are mutually conjugate.

Proof. If $w$ is odd then the group PSL $(2, w)$ contains the group $A_{4}$ as a subgroup (Huppert [10; Hauptsatz II. 8.27]). The group $A_{4}$ contains a Klein four-group $H$ in which the involutions are mutually conjugate (within $A_{4}$ ). Assume $w$ is even; then $w=2^{s}$ with $s \geqq 2$. All the involutions of PSL $\left(2,2^{s}\right)$ are conjugate (Dickson [3; p. 263]). Choosing two nonidentity elements $\sigma, \tau$ in a Sylow 2-subgroup of PSL $\left(2,2^{3}\right)$ gives a Klein four-group $H$ in which the involutions are conjugate (within PSL $(2, w)$ ).

We have the following important result.

THEOREM 3.1. Let $\pi$ be a finite translation plane of dimension $d$ over its kernel $K=G F(q)$, where $q=p^{k}$ with $k \geqq 1$ and $p$ an odd prime. If PSL $(2, w)$, where $w \geqq 3$, is a subgroup of the translation complement $C(\pi)$, then 4 divides the dimension $d$ of the plane $\pi$.

Proof. By Lemma 3.1 and Lemma 3.2 the linear translation complement $L C(\pi)$ contains a Klein four-group $H$ in which the involutions are mutually conjugate within $L C(\pi)$. Thus the theorem follows from Theorem 2.3.

The next theorem considers the case where the characteristic $p$ of the translation plane $\pi$ is equal to 2 .

THEOREM 3.2. Let $\pi$ be a finite translation plane of dimension $d$ over its kernel $K=G F(q)$, where $q=2^{k}$ with $k \geqq 1$. If PSL $(2, w)$, where $w$ is an odd prime power with $w \geqq 7$, is a subgroup of the translation complement $C(\pi)$, then the following statements hold:

(i) Not all the involutions of PSL $(2, w)$ are perspectivities.

(ii) The dimension $d$ of $\pi$ is even.

Proof. First, observe that Lemma 3.1 says $G=$ PSL $(2, w)$ is contained in the linear translation complement $L C(\pi)$. Assume all the involutions of $G$ are perspectivities. Since $G$ fixes the origin $\mathcal{O}$ and since $p=2$, the involutions must be affine elations. By the Hering-Ostrom theorem (Hering [8]), these affine elations generate SL $\left(2,2^{s}\right)$ for some $s \geqq 1$, a Suzuki group $S z\left(2^{r}\right)$ with $r$ odd, an elementary abelian group, or a group of the form $E H$ with $|E|=2$ and $|H|$ odd. But, on the other hand the involutions of $G$ generate $G$ since $G$ is simple $(w>3)$ and $G$ is not one of the four types of 
groups mentioned in the Hering-Ostrom theorem. (Here we need $w>5$ since PSL $(2,5)=\operatorname{PSL}(2,4)=\mathrm{SL}(2,4)$.) Thus we have a contradiction. This proves statement (i) and statement (ii) follows from Lemma 2.14 of Kallaher and Ostrom [15].

REMARK 3.2. The restrictions on $w$ in Theorem 3.2-namely, that $w$ be odd and at least 7-are necessary. First, if $w=5$ or $2^{s} s \geqq 1$ then PSL $(2, w)=$ PSL $\left(2,2^{t}\right)$ for some $t$ and these groups occur in translation planes of odd dimension and even order. Examples include the desarguesian planes of even order. The group PSL $(2,3)=A_{4}$ also occurs in translation planes of odd dimension and even order; examples include semi-field planes of even order.

We can combine Lemma 3.1, Remark 3.1, Theorem 3.2 to give the following information on the existence of translation planes with a collineation group isomorphic to PSL $(2, w)$ for some prime power $w$.

THEOREM 3.3. Let $\pi$ be a finite translation plane of dimension $d$ over its kernel $K=G F(q)$, where $q=p^{k}$ with $k \geqq 1$. If $\pi$ has $a$ collineation group $G=\operatorname{PSL}(2, w)$ with $w \geqq 3$, then one of the following statements holds:

(i) The prime $p$ is odd and 4 divides the dimension $d$.

(ii) The prime $p=2$, the prime power $w$ is odd and at least 7 , and 2 divides the dimension $d$.

(iii) The prime $p=2$ and $w=3,5$, or $2^{s}$ with $s \geqq 2$.

REMARK 3.3. The group PSL $(2,2)=\mathrm{SL}(2,2)=S_{3}$ occurs as the collineation group of many translation planes. For example, if $(Q$, $+, \cdot)$ is a nearfield with an element $a$ of multiplicative order 3 (which happens if and only if $3|(|Q|-1)|$, then in the associated translation plane $\pi$ the involutory affine perspectivities

$$
\begin{aligned}
& (x, y) \longrightarrow(y, x) \\
& (x, y) \longrightarrow\left(y \alpha^{-1}, x a\right)
\end{aligned}
$$

generate a subgroup of $L C(\pi)$ which is isomorphic to $S_{3}$.

We turn now to looking at the possibility of having PSL $(2, w)$ acting on a translation plane under added conditions. We start with the following interesting result.

THEOREM 3.4. Let $\pi$ be a finite translation plane of order $p^{r}$, where $p$ is an odd prime. The translation complement of $\pi$ contains no subgroup $G=$ PSL $\left(2, p^{s}\right)$ with $s>1 / 2 r$.

Proof. Note that the elements of the translation complement 
$C(\pi)$ of $\pi$ on linear transformations of $\pi$ considered as a vector space over $G F(p)$. If $r=1$ then $\pi$ is desarguesian and its translation complement is $\Gamma L(2, p)$ which does not contain a subgroup isomorphic to PSL $\left(2, p^{s}\right)$ for any $s$. Hence we may assume $r>1$.

Assume that $G=\mathrm{PSL}\left(2, p^{s}\right)$ is contained in the translation complement of $\pi$. If $s=r$ then Theorem 4.2.15 of Dembowski [2] implies $\pi$ is desarguesian. By Korollar 2 of Lüneburg [17] the group $G$ cannot fix a line in the projective plane associated with $\pi$. But this contradicts the fact that $G$ fixes $l_{\infty}$. Hence $s \neq r$.

Now, $s>1 / 2 r \geqq 1$; thus, $s \geqq 2$. We have $|G|=\left|\operatorname{PSL}\left(2, p^{s}\right)\right|=$ $1 / 2 p^{s}\left(p^{2 s}-1\right)$. Since $2 s \geqq 4$, the integer $p^{2 s}-1$ has a prime $p$-primitive divisor $u$. (See $[15 ; \S 2 b]$.) This means that $u \mid\left(p^{2 s}-1\right)$ but $u \nmid\left(p^{i}-1\right)$ for all integers $i$ with $1 \leqq i<2 s$. In particular, we must have $u \mid\left(p^{s}+1\right)$. We claim:
(a) $u \nmid\left(p^{r}+1\right)$ and
(b) $u \nmid\left(p^{r}-1\right)$.

Statement (b) follows from the fact that $2 s>r$. For statement (a), note first that if $s>r$ then $u \nmid\left(p^{2 r}-1\right)$ by the definition of a $p$ primitive divisor and hence $u \nmid\left(p^{r}+1\right)$. If $s<r$ then $u \mid\left(p^{r}+1\right)$ would imply $u$ divides $\left(p^{r}+1\right)-\left(p^{s}+1\right)=p^{s}\left(p^{r-s}-1\right)$ and hence $u$ would divide $p^{r-s}-1$. But $r-s<r-1 / 2 r=1 / 2 r<s$ and we have a contradiction to the choice of $u$ as a $p$-primitive divisor of $p^{2 s}-1$. This proves (a).

Finally, note that $u \nmid\left(p^{r-t}-1\right)$ for all $t$ with $0 \leqq t<r$. This follows from the fact that $2 s>r$ and from the definition of a $p$ primitive divisor.

Let $\sigma \in G$ be a collineation of order $u$. Since $u \nmid\left(p^{r}+1\right)$, the collineation $\sigma$ fixes a point $U$ on the line $l_{\infty}$. Consider the component $l=\mathscr{O} U$ of $\pi$. On $l$ the collineation $\sigma$ acts as a linear transformation since $l$ is a subspace of dimension $r$ in $\pi$ considered as a vector space over $G F(p)$. Since $u \nmid\left(p^{r}-1\right)$ the collineation $\sigma$ fixes at least one affine point of $l$ other than the origin $O$. If $\sigma$ did not fix all the affine points of $l$, then its fixed points in $l$ would form a subspace of $l$ having $p^{t}$ points for some $t \geqq 1$. But then $\sigma$ must act as a fixed-point-free permutation on the remaining $p^{r}-p^{t}=$ $p^{t}\left(p^{r-t}-1\right)$ points on $l$; this implies $u \mid\left(p^{r-t}-1\right)$, a contradiction. Thus $\sigma$ fixes all the points of $l$ and hence $\sigma$ is an affine perspectivity. Thus either $u=p$ or $u \mid\left(p^{r}-1\right)$; both possibilities are contradictions. This proves the theorem.

CoRollary 3.4.1. Let $\pi$ be a finite translation plane of dimension $d$ over its kernel $K=G F(q)$, where $q=p^{k}$ with $k \geqq 1$. The translation complement of $\pi$ does not contain a subgroup $G=$ 
PSL $\left(2, q^{t}\right)$ with $t>1 / 2 d$.

Proof. This follows immediately from Theorem 3.4; for $r=k d$ and $t>1 / 2 d$ would imply $k t>1 / 2 k d=1 / 2 r$.

Consider the Lorimer-Rahilly and the Johnson-Walker translation planes of order 16 . In both planes the group $G=\operatorname{PSL}(2,7)$ has the following orbit structure on the line $l_{\infty}$ : The group $G$ fixes pointwise a set $\Delta$ consisting of three points and is transitive on the set $l_{\infty}-\Delta$ consisting of 14 points. This leads to the following definition.

DeFINITION 3.1. A special translation plane is a translation plane of finite order $q^{d}$, where $q=p^{k}$ is the order of the kernel and $d$ is the dimension, possessing a collineation group $G=\operatorname{PSL}(2, w)$ in the linear translation complement and having the following action on $l_{\infty}$, the line at infinity: The group $G$ fixes pointwise a subset $\Delta$ of $l_{\infty}$ with $|\Delta| \geqq 1$ and is transitive on the set $l_{\infty}-\Delta$. The prime power $w$ is called a special invariant of the plane and the subset $\Delta$ is called the associated special set.

We have the following immediate corollary to Theorem 3.1.

THEOREM 3.5. If $\pi$ is a special translation plane of dimension $d$ over its kernel $K=G F(q)$, where $q=p^{k}$ with $k \geqq 1$ and $p$ an odd prime, and if a special invariant of $\pi$ is odd, then 4 divides the dimension $d$ of $\pi$.

Two different viewpoints concerning the set $\Delta$ in Definition 3.1 may be taken. In the Lorimer-Rahilly and Johnson-Walker planes we have

$$
\begin{aligned}
|\Delta| & =3=2+1=p+1 \\
& =\frac{1}{2}(7-1)=\frac{1}{2}(w-1) .
\end{aligned}
$$

This leads to two different, but reasonable, hypotheses that might be added:

$$
\begin{array}{ll}
\text { Hypothesis } J: & |\Delta|=p+1 \\
\text { Hypothesis } K: & |\Delta|=\frac{1}{2}(w-1) .
\end{array}
$$

We investigate Hypothesis $J$ and show that $w \neq p^{s}$ if $p$ is odd.

LEMMA 3.3. Let $\pi$ be a special translation plane of dimension $d$ over its kernel $K=G F(q)$, where $q=p^{k}$ with $k \geqq 1$ and $p$ is an 
odd prime, and assume a special invariant of $\pi$ is the integer $w=$ $p^{s}$ with $s \geqq 1$. If the associated special set $\Delta$ satisfies $|\Delta|=p^{t}+1$ with $t \geqq 1$, then $t \leqq s$ and dk-t divides $2 s$.

Proof. If $|\Delta|=p^{t}+1$ then $\left|l_{\infty}-\Delta\right|=q^{d}-p^{t}=p^{t}\left(p^{d k-t}-1\right)$. Hence $p^{t}\left(p^{d k-t}-1\right)$ divides $\mid$ PSL $\left(2, p^{s}\right) \mid=1 / 2 p^{s}\left(p^{2 s}-1\right)$. Then $p^{t} \mid p^{s}$, which implies $t \leqq s$ and $p^{d k-t}-1$ divides $p^{2 s}-1$ which implies $d k-t$ divides $2 s$.

THEOREM 3.6. Let $\pi$ be a special translation plane of dimension $d$ over its kernel $K=G F(q)$, where $q=p^{k}$ which $k \geqq 1$ and $p$ is a prime, with special invariant $w=p^{s}$ and associated special set $\Delta$. If $|\Delta|=p^{t}+1$ for some $t \geqq 1$ then the characteristic $p=2$.

Proof. Assume $p>2$. By Theorem 3.5 we have $4 \mid r$, where $r=d k$. By Theorem 3.4 the integer $s$ satisfies $s \leqq 1 / 2 r$ and Lemma 3.3 says $t \leqq s$. Hence $t \leqq 1 / 2 r$. Also, Lemma 3.3 says $r-t$ divides $2 s$. Assume $t<1 / 2 r$, and let $2 s=x(r-t)$. If $x>1$, then $r=$ $2 s x^{-1}+t \leqq s+t<r$, a contradiction. Thus, $2 s=r-t$. Now, the group $G=\operatorname{PSL}\left(2, p^{s}\right)$ is transitive on the set $l_{\infty}-\Delta$ and hence, for a point $V \in l_{\infty}-\Delta$ the group $G_{V}$ has order $|G|\left[p^{t}\left(p^{r-t}-1\right)\right]^{-1}=p^{s-t}$. If $\alpha \in G$ is an involution then it must be Baer since it fixes $\Delta$ pointwise and $G$ is simple. But $\alpha$ fixes no point of $l_{\infty}-\Delta$ and hence its fixed subplane has order $p^{t}<p^{s}$, contradicting the fact that $\alpha$ is Baer. This contradiction shows that $t=1 / 2 r$ and $r=2 s$. Thus, $\pi$ is a plane of order $p^{2 s}$ with a subgroup $G=\operatorname{PSL}\left(2, p^{s}\right)$ in its linear translation complement.

Let $\sigma$ be a $p$-element in $G$. By Definition 3.1 the collineation $\sigma$ fixes the $p^{s}+1$ points in $\Delta$. It follows that $\sigma$ fixes pointwise a Baer subplane of $\pi$; that is, the collineation $\sigma$ is a Baer $p$-element in G. By Corollary 4.3 and Proposition 5.1 of Foulser [6] either the Baer $p$-elements of $G$ generate SL $\left(2, p^{s}\right)$ or $p=3$ and $s=1$. Since SL $\left(2, p^{s}\right) \neq \operatorname{PSL}\left(2, p^{s}\right)$ for $p$ odd, we must have $p=3$ and $s=1$. Thus $r=2$, which contradicts the fact that 4 divides $r$.

REMARK 3.4. Not much else can be added to Theorem 3.6. The Hall planes of order $q^{2}$ with $q=2^{k}$ have the collineation group $G=$ $\mathrm{SL}(2, q)=\operatorname{PSL}(2, q)$ and special associated set $\Delta$ with $|\Delta|=q+1$. Since SL $(2, q)$ contains smaller groups of the form SL $\left(2,2^{s}\right)$ with $s \mid k$, we can not prove $t=s$ or $w=q$. We should also remark that in the desarguesian plane of order $q^{3}$, where $q=2^{k}$, the group $G=$ $\operatorname{SL}(2, q)=$ PSL $(2, q)$ is a subgroup of the linear translation complement and $G$ has two orbits on $l_{\infty}$, one of length $q+1$ and a second of length $q^{3}-q$. 
REMARK 3.5. If Hypothesis $K$ is satisfied in a special translation plane, then a simple counting argument shows that $w$ and $p$ are relatively prime. In particular, if in the special translation plane the special set $\Delta$ satisfies

$$
|\Delta|=p+1=\frac{1}{2}(w-1),
$$

then $w=2 p+3$ and an easy calculation shows $w=7$ and $p=2$. The work of Johnson and Ostrom [14] shows that $\pi$ is either the Lorimer-Rahilly plane or the Johnson-Walker plane.

4. The group $\mathrm{SL}(3, w)$ as a collineation group. In this section we consider translation planes having the group SL $(3, w)$, where $w$ is a prime power, as a collineation group. The following result shows that we may in essence restrict ourselves to the linear translation complement.

Lemma 4.1. Let $\pi$ be a finite translation plane of dimension $d$ over its kernel $K=G F(q)$, where $q=p^{k}$ with $k \geqq 1$. If $G=\operatorname{SL}(3, w)$ is a subgroup of the translation complement $C(\pi)$, then $G$ is contained in the linear translation complement $L C(\pi)$.

Proof. Recall that $C(\pi) / L C(\pi)$ is a subgroup of $\Gamma L(2 d, q) / G L(2 d, q)$ which is solvable. Thus $G / G \cap L C(\pi)=G L C(\pi) / L C(\pi)$ is a solvable group and hence $G \cap L C(\pi)$ must be a nonsolvable normal subgroup of $G=\mathrm{SL}(3, w)$. By Aufgabe 6 on p. 135 of Huppert [10] it follows that $G \cap L C(\pi)=G$ and hence $G \leqq L C(\pi)$.

REMARK 4.1. By an argument similar to one exhibited in Remark 3.1 it can be shown that if a translation plane $\pi$ has a collineation group $G=\mathrm{SL}(3, w)$ then its translation complement $C(\pi)$ has $\mathrm{SL}(3, w)$ as a subgroup.

LeMmA 4.2. The group $G=\mathrm{SL}(3, w)$ contains a Klein fourgroup $H$ in which the involutions are mutually conjugate.

Proof. Assume first that $w$ is odd. Let $H$ be the group consisting of the identity matrix and the following three involutory matrices:

$$
A=\left[\begin{array}{rrr}
1 & 0 & 0 \\
0 & -1 & 0 \\
0 & 0 & -1
\end{array}\right] \quad B=\left[\begin{array}{rrr}
-1 & 0 & 0 \\
0 & 1 & 0 \\
0 & 0 & -1
\end{array}\right] \quad C=\left[\begin{array}{rrr}
-1 & 0 & 0 \\
0 & -1 & 0 \\
0 & 0 & 1
\end{array}\right]
$$


It's clear that $H$ is a Klein four-group. Also $A, B, C$ are mutually conjugate; for example, if

$$
D=\left[\begin{array}{lll}
0 & 1 & 0 \\
1 & 0 & 0 \\
0 & 0 & 1
\end{array}\right], E=\left[\begin{array}{lll}
1 & 0 & 0 \\
0 & 0 & 1 \\
0 & 1 & 0
\end{array}\right]
$$

then

$$
D^{-1} A D=B, \quad E^{-1} B E=C, \quad(D E)^{-1} C(D E)=A .
$$

For $w=2$ we have $\operatorname{SL}(3,2)=\operatorname{PSL}(2,7)$ by Satz II.6.14 of Huppert [10]. Lemma 3.2 gives the present lemma in this case. If $w=2^{s}$ with $s \geqq 2$ then SL $\left(3,2^{s}\right)$ contains a subgroup isomorphic to $\mathrm{SL}(3,2)$ and hence the lemma follows in this case also. This proves the lemma.

We come now to our first important result of this section, one which is very similar to Theorems 3.1 and 3.2.

THEOREM 4.1. Let $\pi$ be a finite translation plane of dimension $d$ over its kernel $K=G F(q)$, where $q=p^{k}$ with $k \geqq 1$. If $G=\operatorname{SL}(3, w)$ is a collineation group of $\pi$, then one of the following statements holds:

(i) The prime $p$ is odd and 4 divides the dimension $d$.

(ii) The prime $p=2$, the involutions in $G$ are Baer, and the dimension $d$ is even and at least 4.

Proof. By Lemma 4.1 and Remark 4.1 we may assume $G$ is a subgroup of $L C(\pi)$. If the prime $p$ is odd then statement (i) holds by Lemma 4.2 and Theorem 2.3 .

Assume $p=2$. If one or more involutions in $G$ are perspectivities then they must be affine elations since $G$ fixes the origin $\mathcal{O}$. Furthermore, the normal subgroup generated by these involutions must be $G$ since the center of $G$ has odd order. (See Hilfssatz II.6.2 and Aufgabe 6 on p. 185 of Huppert [10].) The Hering-Ostrom Theorem (Hering [8].) says that either $G$ is solvable, $G$ is a Suzuki group $S_{z}\left(2^{r}\right)$, or $G=\operatorname{SL}\left(2,2^{s}\right)$ for some $s$. But $\mathrm{SL}(3, w)$ is nonsolvable for all $w$; also $\mathrm{SL}(3, w) \neq S_{z}\left(2^{r}\right)$ for any $r$, and $\mathrm{SL}(3, w) \neq$ SL $\left(2,2^{s}\right)$ for any $s$. Hence we have a contradiction. It follows that every involution of $G$ is a Baer involution. By Lemma 2.14 of [15] the dimension $d$ is even. If $d=2$ then Theorem 3.27 of Johnson and Ostrom [13] implies $G=\mathrm{SL}\left(2,2^{s}\right)$ for some $s$, a contradiction. Thus $d>2$. This proves the theorem.

Definition 4.1. An $L R$ translation plane is a finite translation 
$\pi$ having a subgroup $G$ of its linear translation complement $L C(\pi)$ with the following properties:

(i ) $G=S L(3, w)$ for some prime power $w$.

(ii) On $l_{\infty}$, the line at infinity of $\pi$, the group $G$ leaves invariant a set $\Delta$ of order $w+1$ and is transitive on the set $l_{\infty}-\Delta$. The group $G=\mathrm{SL}(3, w)$ is called an $L R$ collineation group and the set $\Delta$ is called the associated $L R$ set.

For the remainder of this section we wish to investigate $L R$ translation planes. Our results will support strongly the conjecture that the only $L R$ translation plane is the Lorimer-Rahilly plane of order 16. To do this we need several lemmas.

LEMMA 4.3. Let $\pi$ be a finite LR translation plane of dimension $d$ over its kernel $K=G F(q)$, where $q=p^{k}$ with $k \geqq 1$. If $G=$ SL $(3, w)$, where $w=p^{t}$ with $t$ a divisor of $k$, is an LR collineation group of $\pi$ with $\Delta$ the associated $L R$ set, then $w=q$ and $d=4$.

Proof. Choose the positive integer $v$ such that $t v=k$; then $q^{d}=w^{v d}$. Letting $u=v d$ gives $q^{d}=w^{u}$. Now $\left|l_{\infty}-\Delta\right|=\left(q^{d}+1\right)-$ $(w+1)=w^{u}-w=w\left(w^{u-1}-1\right)$. Since $G=\mathrm{SL}(3, w)$ is transitive on $l_{\infty}-4$, we have $w\left(w^{u-1}-1\right)$ divides $|\mathrm{SL}(3,4)|=w^{3}\left(w^{3}-1\right)\left(w^{2}-1\right)$. Hence $w^{u}-1$ divides $\left(w^{3}-1\right)\left(w^{2}-1\right)$. Thus there exists an integer $b \geqq 1$ such that

$$
\left(w^{u-1}-1\right) b=\left(w^{3}-1\right)\left(w^{2}-1\right)=w^{5}-w^{3}-w^{2}+1 .
$$

Assume $u \geqq 6$. Then

$$
w^{5}-1 \leqq w^{5}-w^{3}-w^{2}+1
$$

or

$$
w^{2}(w+1)=w^{3}+w^{2} \leqq 2,
$$

a contradiction since $w \geqq 2$. Thus $u \leqq 5$. If $u=v d=5$ then $d$ is odd, contradicting the fact that $d$ is even (Theorem 4.1). Hence $u=v d \leqq 4$. Theorem 4.1 says $d \geqq 4$. Hence $d=4, v=1$, and thus $t=k$, or $w=p^{k}=q$.

LEMMA 4.4. Let $\pi$ be an LR translation plane of dimension $d$ over its kernel $K=G F(q)$, where $q=p^{k}$ with $k \geqq 1$, with the $L R$ collineation group $G=S L(3, q)$ and associated $L R$ set $\Delta$. The group $G$ fixes the set $\triangle$ pointwise.

Proof. If $G$ does not fix $\Delta$ pointwise then $|\Delta| \geqq q^{2}+q+1$ by Mitchell [18] for $q$ odd and Hartley [7] for $q$ even. This a contradiction since $|\Delta|=q+1$. 
LeMma 4.5. Let $w$ be an odd prime power. The group $G=$ $\mathrm{SL}(3, w)$ has an involution $A$ whose centralizer contains a subgroup $G_{1}=\operatorname{SL}(2, w)$.

Proof. Let $A$ be the element of $G$ given in the beginning of the proof of Lemma 4.2. Let $G_{1}$ be the subgroup of $G$ consisting of all matrices of the form

$$
\left[\begin{array}{lll}
1 & 0 & 0 \\
0 & a & b \\
0 & c & d
\end{array}\right]
$$

where the submatrix

$$
\left[\begin{array}{ll}
a & b \\
c & d
\end{array}\right]
$$

is an element of $\operatorname{SL}(2, w)$. Then it is clear that $G_{1}=\operatorname{SL}(2, w)$ and that $G_{1}$ centralizes $A$.

LEMMA 4.6. If $\pi$ is an LR translation plane of dimension $d$ over its kernel $K=G F(q)$, where $q=p^{k}$ with $k \geqq 1$, with the $L R$ collineation group $G=\mathrm{SL}(3, q)$ and associated $L R$ set $\Delta$, then $p=2$.

Proof. Assume $p$ is odd. By Lemma 4.4 the group $G$ fixes $\Delta$ pointwise. Let $\tau$ be the involution of Lemma 4.5 and $G_{1}=\operatorname{SL}(2, q)$ the subgroup of Lemma 4.5. The involution $\tau$ can not be an affine perspectivity since $\tau$ fixes $\Delta$ pointwise. Furthermore, it can not be an homology with axis $l_{\infty}$ since $\left|Z\left(G_{1}\right)\right|=$ g.c.d. $(q-1,3)$. Thus $\tau$ is a Baer involution. Let $\pi_{0}$ be the Baer subplane of $\pi$ fixed pointwise by $\tau$. By Lemma 2.2 the subplane $\pi_{0}$ is a translation plane of dimension at most $1 / 2 d=2$ over its kernel. The group $G_{1}$ fixes $\pi_{0}$ since it centralizes $\tau$; thus $G_{1}$ induces a collineation group $\bar{G}_{1}$ on $\pi_{0}$ and $\bar{G}_{1}=G_{1} / G_{0}$ where $G_{0}$ consists of all elements of $G_{1}$ fixing $\pi_{0}$ pointwise. Note that $\tau \in G_{0}$ and hence $\left|G_{0}\right| \geqq 2$.

We claim that $\bar{G}_{1}=\operatorname{PSL}(2, q)$. First assume that $q>3$. Then the only nontrivial normal subgroups of $G_{1}=\operatorname{SL}(2, q)$ are $G_{1}$ and $Z\left(G_{1}\right)$ which has order 2. Since $G_{0}$ must be solvable (Foulser [5; Theorem 3]), we have $G_{0}=Z\left(G_{1}\right)$ and thus $\bar{G}_{1}=G_{1} / Z\left(G_{1}\right)=\operatorname{PSL}(2, q)$. Assume now that $q=3$. Then $G_{1}=\mathrm{SL}(2,3)$ has four normal subgroups: $1, Z\left(G_{1}\right)$, a quaternion group $Q$, and $G_{1}$. By Theorem 3 of Foulser [5], if $K_{0}$ is the kernel of $\pi_{0}$ then $G_{0}$ is isomorphic to a subgroup of one of the following groups: (a) the additive group $\left(K_{0},+\right)$ of $K_{0}$; (b) the multiplicative group $\left(K_{\infty}^{*} \cdot\right)$ of the kernel $K_{\infty}$ 
of a second Baer subplane; (c) the affine linear group Aff $\left(1, K_{0}\right)$ consisting of all mappings of the form $x \rightarrow x a+b$, where $a, b \in K_{0}$ with $a \neq 0$. It follows that $G_{0}$ has a cyclic Sylow 2-subgroup. Thus $G_{0} \neq Q$ and $G_{0} \neq G_{1}$. Since $\tau \in G_{0}$, it follows that $G_{0}=Z\left(G_{1}\right)$ and thus $\bar{G}_{1}=G_{1} / Z\left(G_{1}\right)=\operatorname{PSL}(2,3)$.

Thus $\pi_{0}$ is a translation plane of dimension 1 or 2 over its kernel having a subgroup $\bar{G}_{1}=\operatorname{PSL}(2, q)$ in its linear translation complement. By Theorem 3.1 the plane $\pi_{0}$ must have dimension at least 4 over its kernel, a contradiction. This contradiction shows that $p=2$.

THEOREM 4.2. Let $\pi$ be a finite $L R$ translation plane of dimension $d$ over its kernel $K=G F(q)$, where $q=p^{k}$ with $k \geqq 1$. If $G=$ $\mathrm{SL}(3, w)$, where $w=p^{t}$ with $t$ a divisor of $k$, is an LR collineation group of $\pi$ with $\triangle$ the associated $L R$ set, then the following statements hold:

(i) The dimension $d=4$ and $w=q$.

(ii) The group $G$ fixes the set $\triangle$ pointwise.

(iii) The prime $p=2$.

Proof. This follows directly from Lemmas 4.3, 4.4, and 4.6.

We come now to the result mentioned after Definition 4.1.

COROLlaRY 4.2.1. Let $\pi$ be a finite translation plane of dimension $d$ over its kernel $K=G F(q)$, where $q=p^{k}$ with $k \geqq 1$. If $G=$ $\mathrm{SL}(3, p)$ is a subgroup of the translation complement $C(\pi)$ which leaves invariant $a$ set $\Delta$ of $p+1$ points on the line at infinity $l_{\infty}$ and is transitive on $l_{\infty}-\Delta$, then $q=p=2$ and $d=4$; furthermore, $\pi$ is either the Lorimer-Rahilly translation plane or the JohnsonWalker translation plane.

Proof. By Lemma 4.1 the plane $\pi$ is an $L R$ translation plane and $G$ is an $L R$ collineation group. By Theorem 4.2 we have $q=$ $p=2$ and $d=4$. It follows that the plane $\pi$ is either the LorimerRahilly plane or the Johnson-Walker plane by the work of Johnson and Ostrom [14].

REMARK 4.2. The results in this section do not completely answer the question: "What translation planes possess a collineation group $G=\mathrm{SL}(3, q)$, where $q$ is the order of the kernel?" They do narrow the investigation down to planes of even order. 


\section{REFERENCES}

1. J. André Über nicht-Desarguessche Ebenen mit transitiver translations-gruppe, Math Z., 60 (1954), 156-186.

2. P. Dembowski, Finite Geometries, Berlin-Heidelberg-New York, Springer Verlag, 1968.

3. L. E. Dickson, Linear Groups, New York, Dover Publications, Inc., 1958.

4. J. D. Dixon, The Structure of Linear Groups, London-Cincinnati-New York, Van Nostrand Reinhold, 1971.

5. D. A. Foulser, Subplanes of partial spreads in translation planes, Bull. London Math. Soc., 4 (1972), 32-38.

6. — Baer p-elements in translation planes, J. Algebra, 31 (1974), 354-366.

7. R. W. Hartley, Determination of the ternary collineation groups whose coefficients lie in $G F\left(2^{n}\right)$, Ann. Math., (2) 27 (1925-26), 140-158.

8. C. Hering, On shears of translation planes, Abh. Math. Sem. Hamb., 37 (1972), 258-268.

9. K. Horfman, and R. Kunze, Linear Algebra, Englewood Cliffs, Prentice-Hall, 1971.

10. B. Huppert, Endliche Gruppen I, Berlin-Heidelberg-New York, Springer-Verlag, 1967.

11. V. Jha, On tangentially transitive translation planes and related systems, Geom. Dedi., 4 (1975), 457-484.

12. N. L. Johnson and T. G. Ostrom, Tangentially transitive planes of order 16, J. Geometry, 10 (1977), 146-163.

13. Translation planes of characteristic two in which all involutions are Baer, J. Algebra, 54 (1978), 291-315.

14. - The translation planes of order 16 that admit PSL (2, 7), J. Combinatorial Series Theory, A 26 (1979), 127-134.

15. M. J. Kallaher and T. G. Ostrom, Collineation groups irreducible on the components of a translation plane, Geometriae Dedicata, in press.

16. P. Lorimer, A projective plane of order 16, J. Combinatorial Series Theory, A 16 (1974), 334-347.

17. H. Lüneburg, Charakterisierung der endlichen desarguesschen projektiven Ebenen, Math. Z., 85 (1964), 419-450.

18. H. H. Mitchell, Determination of ordinary and modular ternary linear groups, Trans. Amer. Math. Soc., 12 (1911), 207-242.

19. T. G. Ostrom, Finite Translation Planes, Lecture Notes in Mathematics, No. 158, Berlin-Heidelberg-New York, Springer-Verlag, 1970.

20. - Finite translation planes, an exposition, Aeq. Math., 15 (1977), 121-133.

21. A. Rahilly, Some translation planes with elations which are not translations, Combinatorial Mathematics III (Proc. Third Austral. Conf., Univ. Queensland, St. Lucia, 1974), p. 197-209. Lecture Notes in Mathematics, No. 452, Berlin-Heidelberg-New York, Springer-Verlag, 1970.

22. M. Walker, $A$ note on tangentially transitive affine planes, Bull. London Math. Soc., 8 (1976), 273-277.

Received August 5, 1980 and in revised form February 25, 1981. The first author was a postdoctoral fellow at Washington State University during the preparation of this article. The second author was supported in part by an NSF grant. He also was a fellow of the Palouse Institute of Scientific Studies and thanks the Institute for providing a wholesome atmosphere.

Glasgow College of TeChNology

Glasgow, Scotland

AND

Washington State University

Pullman, WA 99164 



\title{
PACIFIC JOURNAL OF MATHEMATICS
}

\section{EDITORS}

\author{
DONALD BABBITT (Managing Editor) \\ University of California \\ Los Angeles, CA 90024 \\ Hugo Rossi \\ University of Utah \\ Salt Lake City, UT 84112 \\ C. C. MOORE and ARthur Agus \\ University of California \\ Berkeley, CA 94720
}

J. DUGUNDJI

Department of Mathematics

University of Southern California

Los Angeles, CA 90007

R. FINN and J. MILGRAM

Stanford University

Stanford, CA 94305

\section{ASSOCIATE EDITORS}
R. ARENS
E. F. BeCKENBACH
B. H. NeumanN
F. WoLF
K. YoSHIDA

\section{SUPPORTING INSTITUTIONS}

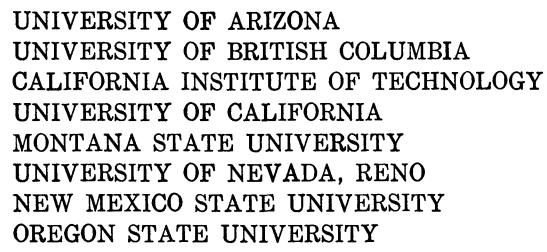

UNIVERSITY OF ARIZONA

UNIVERSITY OF BRITISH COLUMBIA

CALIFORNIA INSTITUTE OF TECHNOLOGY

UNIVERSITY OF CALIFORNIA

MONTANA STATE UNIVERSITY

UNIVERSITY OF NEVADA, RENO

NEW MEXICO STATE UNIVERSITY

OREGON STATE UNIVERSITY

\author{
UNIVERSITY OF OREGON \\ UNIVERSITY OF SOUTHERN CALIFORNIA \\ STANFORD UNIVERSITY \\ UNIVERSITY OF AAWAII \\ UNIVERSITY OF TOKYO \\ UNIVERSITY OF UTAH \\ WASHINGTON STATE UNIVERSITY \\ UNIVERSITY OF WASHINGTON
}

The Supporting Institutions listed above contribute to the cost of publication of this Journal, but they are not owners or publishers and have no responsibility for its content or policies,

Mathematical parers intended for publication in the Pacific Journal of Mathematics should be in typed form or offset-reproduced, (not dittoed), double spaced with large margins. Please do not use built up fractions in the text of the manuscript. However, you may use them in the displayed equations. Underline Greek letters in red, German in green, and script in blue. The first paragraph or two must be capable of being used separately as a synopsis of the entire paper. Please propose a heading for the odd unmbered pages of less than 35 characters. Manuscripts, in triplicate, may be sent to any one of the editors. Please classify according to the scheme of Math. Reviews, Index to Vol. 39. Supply name and address of author to whom proofs should be sent. All other communications should be addressed to the managing editor, or Elaine Barth, University of California, Los Angeles, California, 90024.

50 reprints to each author are provided free for each article, only if page charges have been substantially paid. Additional copies may be obtained at cost in multiples of 50 .

The Pacific Journal of Mathematics is issued monthly as of January 1966, Regular subscription rate: $\$ 114.00$ a year (6 Vol., 12 issues). Special rate: $\$ 57.00$ a year to individual members of supporting institution.

Subscriptions, orders for numbers issued in the last three calendar years, and changes of address shoud be sent to Pacific Journal of Mathematics, P.O. Box 969, Carmel Valley, CA 93924, U.S.A. Old back numbers obtainable from Kraus Periodicals Co., Route 100, Millwood, NY 10546.

PUBLISHED BY PACIFIC JOURNAL OF MATHEMATICS, A NON-PROFIT CORPORATION

Printed at Kokusai Bunken Insatsusha (International Academic Printing Co., Ltd.). 8-8, 3-chome, Takadanobaba, Shinjuku-ku, Tokyo 160, Japan. 


\section{Pacific Journal of Mathematics}

\section{Vol. 103, No. $2 \quad$ April, 1982}

Alberto Alesina and Leonede De Michele, A dichotomy for a class of positive

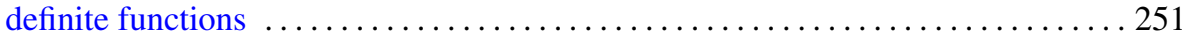

Kahtan Alzubaidy, Rank 2 -groups, $p>3$, and Chern classes . . . . . . . . . . 259

James Arney and Edward A. Bender, Random mappings with constraints on

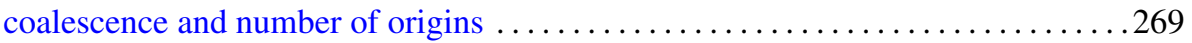

Bruce C. Berndt, An arithmetic Poisson formula . . . . . . . . . . . . . . . 295

Julius Rubin Blum and J. I. Reich, Pointwise ergodic theorems in 1.c.a. groups . . . 301

Jonathan Borwein, A note on $\varepsilon$-subgradients and maximal monotonicity . . . . . . . 307

Andrew Michael Brunner, Edward James Mayland, Jr. and Jonathan Simon,

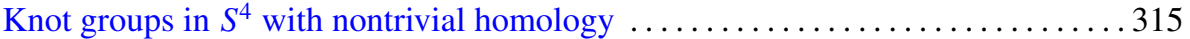

Luis A. Caffarelli, Avner Friedman and Alessandro Torelli, The two-obstacle

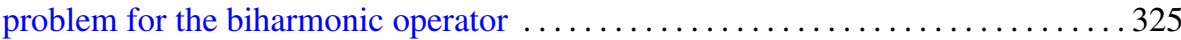

Aleksander Całka, On local isometries of finitely compact metric spaces . . . . . . 337

William S. Cohn, Carleson measures for functions orthogonal to invariant

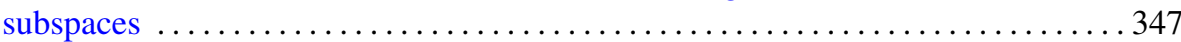

Roger Fenn and Denis Karmen Sjerve, Duality and cohomology for one-relator

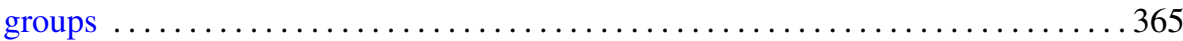

Gen Hua Shi, On the least number of fixed points for infinite complexes . . . . . . . 377

George Golightly, Shadow and inverse-shadow inner products for a class of linear

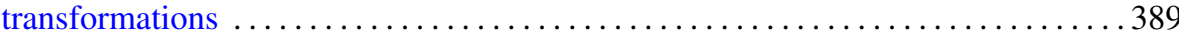

Joachim Georg Hartung, An extension of Sion's minimax theorem with an

application to a method for constrained games $\ldots \ldots \ldots \ldots \ldots \ldots \ldots \ldots \ldots \ldots . \ldots 4$

Vikram Jha and Michael Joseph Kallaher, On the Lorimer-Rahilly and

Johnson-Walker translation planes

Kenneth Richard Johnson, Unitary analogs of generalized Ramanujan sums .

Peter Dexter Johnson, Jr. and R. N. Mohapatra, Best possible results in a class of inequalities

Dieter Jungnickel and Sharad S. Sane, On extensions of nets

Johan Henricus Bernardus Kemperman and Morris Skibinsky, On the

characterization of an interesting property of the arcsin distribution ...

Karl Andrew Kosler, On hereditary rings and Noetherian $V$-rings

William A. Lampe, Congruence lattices of algebras of fixed similarity type. II . . . . 475

M. N. Mishra, N. N. Nayak and Swadeenananda Pattanayak, Strong result for real zeros of random polynomials

Sidney Allen Morris and Peter Robert Nickolas, Locally invariant topologies on free groups

Richard Cole Penney, A Fourier transform theorem on nilmanifolds and nil-theta functions

Andrei Shkalikov, Estimates of meromorphic functions and summability theorems

László Székelyhidi, Note on exponential polynomials

William Thomas Watkins, Homeomorphic classification of certain inverse limit spaces with open bonding maps $\ldots \ldots \ldots \ldots \ldots \ldots \ldots \ldots$

David G. Wright, Countable decompositions of $E^{n}$

Takayuki Kawada, Correction to: "Sample functions of Pólya processes" .

Z. A. Chanturia, Errata: "On the absolute convergence of Fourier series of the 